Revista de Derecho YACHAQ

ISSN: 1817-597x (impresa) / ISSN: 2707-1197 (en linea)

Centro de Investigación de los Estudiantes de Derecho (CIED)

Universidad Nacional de San Antonio Abad del Cusco

N. ${ }^{\circ} 10-2019$

[pp. 109-116]

Fecha de recepción: 05/08/19

Fecha de aceptación: 17/09/19

\title{
Economía colaborativa, subordinación económica y el puesto de trabajo del futuro
}

\author{
Collaborative Economy, Economic Subordination \\ and the Job Position of the Future
}

\author{
Willy Monzón Zevallos ${ }^{[*]}$
}

\begin{abstract}
RESUMEN: la prestación de servicios a través de plataformas digitales evidencia nuevas formas de trabajo atípicas, descentralizadas y flexibles en un sistema de economía colaborativa bajo subordinación económica, que no se enmarcan en el concepto tradicional de trabajo objeto de protección del derecho laboral; como la tecnología modificó la actividad productiva a partir de un cambio económico y social, resulta necesario evaluar si se requiere una nueva regulación que permita la adaptación del derecho del trabajo a esta realidad compleja; para la OIT, no queda claro, qué fracción de la fuerza de trabajo mundial acabará representando el trabajo virtual y no se sabe si estas formas de trabajo acabarán entrando en la esfera de la relación laboral, si se convertirán en nuevos tipos de trabajo informal o si no podrán encajar en los marcos normativos existentes; ante ello, es necesario analizar cuál sería la forma de protección a otorgar a los prestadores de servicios en un sistema de economía colaborativa subordinados económicamente y si ello, implicaría modificar sustancialmente el concepto clásico del derecho del trabajo en el futuro
\end{abstract}

ABSTRACT: the provision of services through digital platforms, demonstrates new atypical, decentralized and flexible forms of work in a collaborative economy system under economic subordination, which are not framed in the traditional concept of work subject to labor law protection; as technology modified the productive activity from an economic and social change, it is necessary to evaluate whether a new regulation is required that allows the adaptation of the right to work to this complex reality; for the ILO, it is not clear that fraction of the global workforce will end up representing virtual work and it is not known whether these forms of work will end up entering the sphere of the labor relationship, if they will become new types of informal work or if they cannot fit into existing regulatory frameworks; given this, it is necessary

[*] Asociado activo de la Sociedad Peruana de Derecho del Trabajo y de la Seguridad Social, abogado experto en derecho laboral y seguridad social, socio senior del área laboral y previsional de Estudio Muñiz, Olaya, Meléndez, Castro, Ono \&amp; Herrera. Contacto:wmonzon@munizlaw.com 
to analyze the form of protection to be granted to service providers in an economically subordinated collaborative economy system and if this would imply substantially modifying the classic concept of labor law in the future

PALABRAS CLAVE: derecho del trabajo, prestadores de servicios, economía colaborativa, plataformas digitales, subordinación económica.

KEY WORDS: labour law, service providers, collaborative economy, digital platforms, economic subordination.

\section{INTRODUCCIÓN}

La prestación de servicios a través de plataformas digitales pone en evidencia nuevas formas de trabajo atípicas descentralizadas y flexibles, en el sistema de economía colaborativa bajo subordinación económica, que no se enmarcan en el concepto tradicional de trabajo objeto de protección del derecho laboral; el desarrollo tecnológico, modificó la actividad productiva a partir de un cambio económico y social, siendo necesario evaluar si se requiere una nueva regulación que permita la adaptación del derecho del trabajo a esta realidad compleja.

\section{EL TRABAJO PROTEGIDO POR EL DERECHO LABORAL}

Teniendo en cuenta lo manifestado por Ramírez Bosco (2009); Deveali, fue quien señaló que «definir los límites del contrato de trabajo es algo que se debe hacer para determinar el ámbito de aplicación dentro del cual es necesario, posible y se justifica una protección especial» (p. 235), es así que, la dependencia bajo subordinación jurídica del prestador de servicios se constituyó en el factor determinante para la existencia de un contrato de trabajo, siendo los servicios que se prestan en esa condición los que requieren la protección especial del derecho laboral.

De acuerdo a Ugarte Cataldo (2011), en el origen de la legislación social —antecedente inmediato del Derecho del Trabajo - no aparece nada ni en las normas ni en los que hablan sobre las normas que se llame subordinación o dependencia jurídica; es así que se formula la siguiente pregunta: ¿cómo llega, en ese escenario, la subordinación a transformarse en la noción cardinal del Derecho del Trabajo? La respuesta del mismo autor es sencilla, al precisar que la subordinación no llega, sino que nace con el Derecho del Trabajo. Mientras no aparece aquella tampoco existe aquel (p. 21).

Para Ermida Uriarte y Hernández Álvarez (2000), el concepto de subordinación como factor fundamental para determinar el ámbito de aplicación del derecho del trabajo fue propuesto por Ludovico Barassi, quien definió la subordinación como la sujeción plena y exclusiva del trabajador al poder directo y de control del empleador. Sin embargo, estos autores consideran que, para aplicar el derecho del trabajo, también resulta determinante que exista: i) ajenidad, ii) la inserción del trabajador en la organización empresarial, iii) prestación de servicios bajo subordinación económica o bajo parasubordinación, y de ser el caso, iv) autonomía colectiva.

Por su parte, Supiot (2008) considera que subordinado es aquel que se halla sometido a una orden. Sin embargo, esto no dice nada acerca de la naturaleza de dicha orden. La orden puede ser la estructura que identifica una organización y que le permite perdurar en su seno. Pero la orden puede ser también el mando, la expresión de una voluntad que se impone a otro. En el primer sentido, la subordinación designa un vínculo de pertenencia; en el segundo, un vínculo de obediencia. El contrato de trabajo fue siempre el punto de tensión entre estos dos aspectos de la subordinación (pp. 70-71).

Por tanto, con relación a la subordinación es importante tener en cuenta que, a criterio del autor citado en el párrafo anterior, este elemento consigna: i) un vínculo de dependencia (subordinación funcional resultante de la integración a una organización); y ii) un vínculo de obediencia (subordinación personal resultante de la sumisión a otro). En consecuencia, es a partir del vínculo de obediencia que nace la subordinación jurídica la que caracteriza al asalariado por su sumisión personal a las órdenes y directivas del empleador (Supiot, 2008, p. 72). 
A partir de la subordinación jurídica, debemos analizar si actualmente se pueden considerar las nuevas formas de prestación de servicios ejecutadas a través de plataformas digitales bajo subordinación económica, como prestaciones objeto de protección por el derecho del trabajo, determinando los casos en que nos encontramos frente a indicios que nos permitan concluir qué servicios no son en realidad de naturaleza independiente y si es posible extender la protección del derecho del trabajo a servicios que no se enmarcan en la definición tradicional del trabajo dependiente.

De otro lado, la subordinación económica se configura por la condición de inferioridad del trabajador frente a quien requiere sus servicios, sean dependientes o independientes; sin embargo, esa figura es económica más no jurídica; por lo que, incluso, un trabajador autónomo, cuyos ingresos en mayor porcentaje provengan de una sola relación contractual, podría considerarse como dependiente económicamente, supuesto que se advierte actualmente en las prestaciones de servicios autónomos ejecutados a través de plataformas digitales.

Para Palomeque (2011), la actual situación económica y social huye, ciertamente, de un modelo único de relación de trabajo, para reclamar los cambios institucionales que permitan la incorporación de diversas formas de organización de la producción y del trabajo existentes. En este contexto, se ha abierto camino de modo incuestionable el recurso a la promoción y desarrollo del trabajo autónomo y, por lo que ahora interesa, las propuestas del campo de aplicación del Derecho del Trabajo a los trabajadores autónomos, en general o tan solo aquellos que, sin poder ser calificados lógicamente de asalariados, se encuentran, sin embargo, en una situación de dependencia económica frente al empresario o cliente principal [parasubordinación del trabajador o trabajo subordinado] (p.151).

El autor citado considera que a partir del incremento del trabajo autónomo resulta necesario garantizar a aquellos trabajadores niveles de protección social semejantes a los que asisten a trabajadores asalariados, pero en el caso de los primeros, únicamente a partir de la existencia de dependencia económica frente a quien los contrata, lo que significa extender la protección del derecho del trabajo a actividades que tradicionalmente se encontraban excluidas de aquel ámbito, lo que representaría la mutación del derecho del trabajo tradicional.

Al existir servicios que se prestan fuera del concepto tradicional de subordinación, la regulación actual resultaría insuficiente para atender las nuevas formas de trabajo que se originan a partir de los avances tecnológicos, principalmente, aquellas que se ejecutan a través de plataformas digitales; por lo que, a criterio de Supiot citado por Ameglio, «la noción de subordinación ya no permite abarcar la diversidad de formas que reviste el trabajo por cuenta ajena, por lo que tal vez habría que establecer un nuevo régimen jurídico para las personas que trabajan que rebase los límites actuales del trabajo asalariado» (p. 224).

Los autores referidos consideran que el derecho del trabajo es el instrumento jurídico que regula todo trabajo humano; pese a que este derecho se origina en la prestación de servicios dependientes; otro grupo considera que es necesario que el derecho laboral abarque la prestación de servicios que se ubica en medio del trabajo dependiente e independiente; entre los que sostienen tal posición, encontramos a Córdova, Goldin y Feldman, quienes proponen crear una categoría que abarque el trabajo no tradicional, otorgando en esos casos un mínimo de protección.

Muchas de las nuevas formas de trabajo se ejecutan por prestadores autónomos que se encuentran bajo dependencia económica de quien requiere sus servicios, los que pueden ser calificados como parasubordinados o casi subordinados, siendo complicado determinar si aquellos únicamente a partir del factor económico tendrían la protección del derecho laboral, ya que el elemento que determina la diferencia sustancial y característica entre el trabajo dependiente con el independiente es la subordinación jurídica que origina la existencia del contrato de trabajo.

Pese a que en la realidad se prestan servicios que no generan vínculo laboral de acuerdo al concepto tradicional del derecho del trabajo, actualmente no se modificó la definición de subordinación; por lo que, a la fecha respecto a los trabajadores autónomos económicamente dependientes que prestan servicios en plataformas digitales, no 
existe consenso para que aquellos ingresen dentro del ámbito de protección del derecho laboral, mucho menos cuentan con un marco normativo que les reconozca una mínima protección.

\section{ECONOMÍA COLABORATIVA Y PLATAFOR- MAS DIGITALES}

Como consecuencia del desarrollo tecnológico, se hizo usual, cotidiano y necesario el uso de la internet, para ofertar y requerir bienes y servicios a través del uso de plataformas que han digitalizado la economía, dinamizando los negocios al actuar como intermediarias entre clientes, vendedores y prestadores de servicios a través de modelos de negocios menos costosos, más flexibles y descentralizados, los que se desarrollan por medio de Apps, todo ello en un sistema de economía colaborativa y un sistema de economía bajo demanda.

De acuerdo a Tom Slee (2016), «los partidarios de la economía colaborativa la describen como una nueva clase de negocio, y otros como un movimiento social» (p.13), todo a partir del uso de plataformas tecnológicas; Rodríguez Gonzales (2018) precisa que «la Comisión Nacional de los Mercados y la Competencia de España, entiende esta forma de negocio como un nuevo modelo de consumo basado en el intercambio de particulares, de bienes y servicios que permanecían ociosos o infrautilizados a cambio de una compensación pactada entre las partes» (p. 4).

La Comisión Europea (2016) considera a la economía colaborativa como un «modelo de negocio en los que se facilitan actividades mediante plataformas colaborativas que crean un mercado abierto para el uso temporal de las mercancías o servicios ofrecidos a menudo por particulares» ( $p$. 3). Pese a ello, en la realidad, la oferta de bienes o servicios en este sistema no solo comprende el uso temporal de bienes infrautilizados, sino también la prestación cotidiana de servicios no temporales, siendo claros ejemplos de lo manifestado Airbnb, Uber, Glovo, Deliveroo y entre otras plataformas.

Adicionalmente, también tenemos la economía bajo demanda (on demand economy) que se refiere a aquellos modelos de consumo y provisión de servicios que se basan en la intermediación entre la oferta y la demanda generada habitualmente de profesional a consumidor (B2C) a través de plataformas digitales que no prestan el servicio subyacente y cuya prestación se origina en base a las necesidades del usuario que demanda y se adapta a sus preferencias, prestándose normalmente a cambio de una contraprestación y habitualmente con ánimo de lucro (Rodriguez Marín).

Dentro de este modelo, como refiere Todolí (2017), se pueden observar hasta cuatro tipos de negocios: el Crowdsorcing online (permite conectar un gran número de trabajadores y distribuir tareas para ser ejecutadas por ordenador) vs. Crowdsorcing offline (permite conectar un gran número de trabajadores para realizar tareas de manera física) y el Crowdsorcing genérico vs. Crowdsorcing específico (p. 22). Siendo importante precisar que todos estos tipos de negocios se caracterizan por el uso de la internet y las plataformas digitales.

El mismo autor, citando a Howe, precisa que el Crowdsourcing o Crowdwork consiste en tomar una prestación de un servicio tradicionalmente realizada por un trabajador, y descentralizarla hacia un indefinido y, normalmente gran número de personas en forma de llamamiento o convocatoria. Debemos diferenciar los servicios prestados a través de las plataformas digitales bajo los modelos descritos, de los servicios ejecutados por medio del teletrabajo, ya que este se realiza bajo una relación de dependencia y subordinación propia del derecho laboral.

Adicionalmente, otra diferencia entre el teletrabajo y el Crowdsourcing es que en este modelo las prestaciones las ejecutan trabajadores autónomos; se debe tener en cuenta que el trabajo objeto de protección por el derecho laboral fue aquel definido antes de la aparición de las plataformas digitales, lo que genera la necesidad de analizar los modelos económicos referidos, con el objeto de establecer si en efecto el uso de aquellas plataformas solamente implica una relación de intermediación y no genera prestación de servicios bajo dependencia de manera encubierta.

El debate se originó con el caso de Uber, que es la plataforma virtual intermediaria que conecta a través de una aplicación móvil a clientes que requieren un servicio de traslado (transporte) con conductores registrados en su base de datos, otorgando 
además de una tarifa inferior a la de un servicio de taxi convencional, beneficios como conocer al conductor, su calificación, su vehículo, el tiempo del traslado, obteniendo Uber a cambio de permitir el uso de su plataforma el pago de una comisión por cada viaje realizado.

Si bien es importante destacar que este servicio de transporte se presta en el tiempo y las oportunidades que determine el conductor afiliado a la plataforma y no Uber, en base a la descripción realizada en el párrafo anterior, el servicio de traslado que oferta esa empresa, inicialmente, no podría ser considerado como uno que genere relación de dependencia y subordinación entre la plataforma y los conductores afiliados, ya que estaríamos frente al típico caso de la prestación de un servicio bajo subordinación económica.

Pese a ello, como refiere Aragüez Valenzuela (2017), para ser conductor de Uber existía un procedimiento de evaluación que incluía una entrevista personal y, además, se exigían requisitos indispensables, entre ellos, contar con su propio vehículo de menos de diez años de antigüedad, licencia de conducir vigente y, en caso el candidato fuera seleccionado, firmaba un acuerdo de colaboración en el cual se especificaban las condiciones del servicio, así como la forma del reparto de los ingresos e, incluso, se le proporcionaba recomendaciones y un Iphone con la App de Uber (p. 7).

A partir de las condiciones en las cuales se presta el servicio en la plataforma de propiedad de Uber, las recomendaciones que se brindaba a los conductores, como por ejemplo, vestir en forma profesional, abrir la puerta al cliente, contar con paraguas, entre otras y la valoración que se efectúa a la calificación de los conductores que realizan los clientes, que al ser negativa puede generar la baja de la plataforma, el Tribunal de Justicia Europeo (2017) consideró que el servicio de transporte que oferta Uber a través de conductores no profesionales no se limita a un servicio de intermediación.

Es evidente que no existe un criterio unánime que permita identificar a los conductores de esta plataforma como trabajadores dependientes; sin embargo, pese a la inexistencia de un horario, a partir de un análisis de los rasgos de laboralidad en la prestación de los servicios, tales como la existencia de un procedimiento de selección, el otorgar una herramienta de trabajo, fijar la tarifa por el servicio, sancionar el rechazo de clientes y la posibilidad de dar de baja al conductor por malas valoraciones, se configurarían indicios de servicios prestados bajo subordinación jurídica.

Es importante precisar que la plataforma virtual (App) al ser de propiedad de una empresa con una marca con reputación conocida determina que el servicio se preste bajo ajenidad, hecho que, sumado a los indicios de laboralidad, determinarían la existencia de un contrato de trabajo. A partir de ello, Todolí (2017) considera que nos encontramos ante una forma nueva de dependencia aplicable a los modelos de economía de plataformas digitales (p. 63) y propone una lista de indicios de laboralidad para determinar si estamos ante una simple intermediación o frente a una relación de trabajo.

Es evidente que nos encontramos ante un nuevo tipo de trabajo y trabajador que interactúan en sistemas de negocios no tradicionales, es así que, a decir de Molina (2017), estas tendencias en la organización de la producción y del trabajo cuestionan abiertamente la relación laboral en su configuración clásica como instrumento jurídico principal para articular el intercambio de trabajo por retribución (p. 411). Es por ello que resulta necesario evaluar si el derecho del trabajo abarca o no a estas nuevas formas de prestación de servicios.

Teniendo en cuenta que a partir del crowdworking se prestan servicios a través de la intermediación de las plataformas digitales, cuyos propietarios ejercen total control del negocio, fragmentando los puestos de trabajo, desmantelando los salarios en micropagos, desplazando al trabajador subordinado, eliminando sus derechos laborales y previsionales; por tanto, resulta necesario definir si la prestación de servicios bajo dependencia económica genera la necesidad de regular esta nueva forma de organización del trabajo en plataformas digitales.

Si no se regulan las actividades que se desarrollan a través de plataformas digitales, como dice Vallecito (2017), «se excluye de las relaciones laborales sustituyéndolas por el trabajo por cuenta propia» (p. 464), generándose un desplazamiento del trabajo dependiente al trabajo autónomo; por lo que resulta necesario establecer cuando nos en- 
contramos frente a plataformas digitales (Apps) que actúan como simples intermediarias o cuando estamos frente a empresas tecnológicas que participan en forma directa en la prestación del servicio.

Si bien las normas laborales solo regulan la relación de trabajo tradicional, es decir, aquella en la que se ejecutan prestaciones de servicios subordinados jurídicamente, aplicando el principio de veracidad (primacía de la realidad) ante la existencia de indicios de laboralidad, es posible llegar a establecer qué servicios prestados a través de plataformas digitales se ejecutan bajo ajenidad y dependencia; a partir de lo cual los trabajadores deben ser objeto de protección del derecho del trabajo y del derecho de la seguridad social.

Sin perjuicio de lo manifestado, el Informe Inicial para la Comisión Mundial sobre el Futuro del Trabajo (2017), elaborado por la OIT, precisó que los cambios tecnológicos y la digitalización están creando nuevas formas de trabajo invisible, como el «trabajo virtual» o el «trabajo digital». En estas nociones se incluyen actividades de la economía de ocupaciones transitorias o de colaboración abierta (crowdsourcing), entre ellas, las microtareas, las actividades de asistencia virtual, o una amplia gama de tareas que sustentan las actividades de las redes sociales.

El informe considera errado creer que las nuevas tecnologías y los algoritmos son los que procesan la información que se recoge mediante esas tecnologías de la información y comunicación. En realidad, son los «trabajadores invisibles» (sin ubicación propia ni relación de empleo reconocida); por lo que no queda claro qué fracción de la fuerza de trabajo mundial acabará representando el trabajo virtual, y si estas formas de trabajo acabarán entrando en la esfera de la relación laboral, si se convertirán en nuevos tipos de trabajo informal o si no podrán encajar en los marcos normativos existentes ${ }^{[1]}$.

Pese a la falta de reglamentación de esos servicios, a la fecha ya existen algunos pronunciamientos a nivel de inspección de trabajo y tribuna- les respecto de la naturaleza dependiente de los servicios prestados por trabajadores a través de plataformas digitales, es el caso de Uber, en el que la inspección de trabajo de Cataluña, el 2015, concluyó que los conductores de esta plataforma (Uber Systems Spain) eran sus trabajadores (Gozzer, 2015), similar pronunciamiento fue el de la inspección de trabajo de Valencia, que en el 2018, luego de una investigación, concluyó que los repartidores de Glovo eran falsos autónomos.

La inspección de trabajo consideró que los repartidores de Glovo no eran trabajadores autónomos al concurrir los presupuestos constitutivos del contrato de trabajo. Pese a ello, los pronunciamientos judiciales no son uniformes, un juez de Valencia determinó que un repartidor de Deliveroo era un falso autónomo y un juez de Madrid concluyó que un repartidor de Glovo era un trabajador por cuenta propia. Pese a ello, como refiere Alvaréz (2018), en Estados Unidos y Gran Bretaña, se propuso crear una categoría intermedia entre autónomo e independiente, denominada «non-employee workers» (p. 3).

Los resultados de la inspección de trabajo de Valencia en el caso de los riders de Deliveroo, que fueron considerados trabajadores por cuenta ajena, generaron un proceso judicial en el que, el Juzgado de lo Social 5 de Valencia, por sentencia de fecha 27 de junio de 2019, falló a favor de noventa y siete repartidores considerándolos falsos autónomos, al existir indicios de laboralidad y, además, porque el medio de producción de los repartidores es la plataforma digital de propiedad de la empresa, sin la cual no es posible que se ejecute la prestación del servicio (Lopez Ahumada, 2019, p. 26).

De igual forma, repartidores de Glovo fueron declarados trabajadores dependientes de la empresa en un proceso judicial, a través de la sentencia dictada por el Juzgado de lo Social 1 de Gijón, la misma que fue ratificada por la Sala de lo Social del Tribunal Superior de Justicia de Asturias por sentencia del 25 de julio de 2019, siendo esta la

[1] Informe Inicial para la Comisión Mundial sobre el Futuro del Trabajo. Recuperado de: https://www.ilo.org/ wcmsp5/groups/public/---dgreports/---cabinet/documents/publication/wcms_591504.pdf

(consultado en mayo de 2019). 
primera que dicta un Tribunal Superior en atención a las notas de ajenidad y dependencia, propias de quien se encuentra dentro del ámbito de dirección de una empresa.

\section{EL PUESTO DE TRABAJO DEL FUTURO}

Teniendo en cuenta el desarrollo vertiginoso de los avances tecnológicos en todos los ámbitos del quehacer humano, queda claro que las formas de trabajo en el futuro se verán modificadas cada vez más por la automatización y el uso de las plataformas digitales (Apps), lo que generará que muchas formas de prestación de servicios, en atención a sus características y por la forma en que se ejecuten, resultarán atípicas y no se enmarcarán en el concepto tradicional de dependencia que genera la protección especial del derecho del trabajo.

Respecto la automatización, Oppenheimer (2018) refiere el estudio publicado en el 2013 por Carl Benedikt Frey y Michael A. Osborne (investigadores de la Oxfort Martin School) en el que pronosticaron que el $47 \%$ de los empleos podrían desaparecer en los próximos 15 o 20 años por la automatización (pp. 15 y ss.), a partir de la revolución robótica y la inteligencia artificial, lo que eliminaría decenas de millones de empleos, ello por el avance tecnológico cada vez más acelerado; el mismo autor, en base al algoritmo de Frey y Osborne refiere un ranking de los trabajos más amenazados.

Si bien las formas de trabajo cada vez se alejan más de aquellos modelos tradicionales que generaron el nacimiento del derecho laboral, como mecanismo para otorgar protección especial a quien ofrecía su fuerza de trabajo a cambio de una remuneración; pese a que los medios de producción o los sistemas económicos pueden cambiar; se debe tener en cuenta que el concepto de subordinación jurídica seguirá siendo el elemento principal y característico que determinará cuándo estamos ante una relación de trabajo dependiente.

Por tanto, si bien en el puesto de trabajo del futuro se realizará la prestación de servicios a través de procesos de producción tecnológicos, automatizados y en plataformas digitales, sujetos a subordinación económica, en caso se presenten indicios tradicionales de laboralidad, estaremos ante una categoría intermedia entre el trabajo dependiente e independiente; en consecuencia, en ese contexto, resulta necesario evaluar la necesidad de regular esta forma de prestación de servicios, ello con el objeto de otorgar protección a esos trabajadores.

\section{v. CONCLUSIONES}

La evolución tecnológica, el uso de la internet y las plataformas digitales para prestar servicios generan nuevas formas de trabajo y sistemas productivos que no se enmarcan dentro del concepto tradicional de dependencia regulado por el derecho laboral, quedando así fuera del ámbito de protección de este derecho, ya que no se presenta la subordinación jurídica, principal elemento que genera la existencia del contrato de trabajo.

A través de las plataformas digitales, en el sistema de economía colaborativa se ofertan servicios que se ejecutan por trabajadores autónomos, con libertad para determinar cómo y cuándo prestarán los servicios; sin embargo, también los propietarios de las plataformas pueden intervenir en el servicio; por lo que nos encontramos ante una realidad que evidencia independencia y a la vez dependencia, lo que demuestra la existencia de una categoría de trabajador que no se encuentra regulada.

La subordinación económica no podría generar por si sola que un trabajador autónomo se encuentre protegido por el derecho laboral; por lo que resulta necesario analizar en cada caso si en la prestación de servicios a través de plataformas digitales se presentan indicios de laboralidad, a partir de los cuales se pueda advertir la naturaleza no autónoma de la prestación de servicios y, por consiguiente, el derecho a ser reconocido como trabajador dependiente.

Si bien la automatización y la tecnología modificarán los sistemas productivos ahora y en el futuro, la subordinación jurídica como principal elemento del contrato de trabajo permanece inmutable; por lo que, incluso, frente a los cambios que se presenten, si el servicio en plataformas se presta bajo dependencia, ajenidad, dirección, fiscalización e inmerso en la organización de la empresa sin autonomía siempre estaremos ante un contrato de trabajo que deberá ser objeto de protección por el derecho laboral. 


\section{REFERENCIAS BIBLIOGRÁFICAS}

Álvarez Cuesta, H. (2018). La Gig Economy y la obligación de coordinar la seguridad y salud de sus autónomos. En Impacto Laboral en las redes empresariales. Granada: Comares.

Ameglio, E. J. (s.f.). La subordinación ¿sigue siendo la nota que caracteriza al contrato de trabajo? En La Relación de Trabajo.

Araguez Valenzuela, L. (Enero-marzo de 2017). Nuevos modelos de la economía compartida: Uber Economy como plataforma virtual de prestación de servicios y su impacto en las relaciones laborales. Revista Internacional de Relaciones Laborales y Derecho del Empleo, Vol. 5 (1).

COM. (2016).

Ermida Uriarte, O. y Hernández Álvarez, O. (s.f.). Crítica a la subordinación. Recuperado de: http:// biblio.juridicas.unam.mx/libros/3/1090/16.pdf

Gozzer, S. (2015). Trabajo dice que los chóferes de Uber son empleados de la firma. La Inspección concluye que debería haberles dado de alta en la Seguridad Social. Recuperado de: https://elpais.com/economia/2015/06/12/actualidad/1434135569_865496.html

Lopez Ahumada, E. (Junio de 2019). La declaración de relación laboral de los "riders" en el caso Deliveroo. Actualidad Laboral. Recuperado de: https://revista-actualidadlaboral.com/revistaactualidad-laboral-junio-2019/

Molina Navarrete, C. (2017). Dereho y Trabajo en la Era Digital: ¿Revolución Industrial 4.0 o Economía Sumergida 3.0? En S. D. Social (Ed.). Conferencia Nacional Tripartita: El Futuro del Trabajo que queremos. Vol. 2. Palacio de Zurbano, Madrid. Recuperado de: https://www. ilo.org/wcmsp5/groups/public/---europe/---rogeneva/---ilo-madrid/documents/publication/ wcms_615487.pdf.

Oficina Internacional del Trabajo. (2017). Informe Inicial para la comisión Mundial sobre el Futuro del Trabajo. Ginebra. Recuperado de: https://www.ilo.org/wcmsp5/groups/public/--dgreports/---cabinet/documents/publication/ wcms_591504.pdf
Oppenheimer, A. (2018). iSálvese quien pueda! EI futuro del trabajo en la era de la automatización. 1. ${ }^{a}$ ed. México: Penguin Random House Group.

Palomeque López, C. M. (2011). El trabajo Autónomo. En El Trabajo. Madrid: Universitaria Ramón Areces.

Ramírez Bosco, L. (2009). Sobre los recurrentes problemas de la dependencia laboral. En: La relación de trabajo. 1. ${ }^{a}$ ed. Buenos Aires: Rubinzal Culzoni Editores.

Rodriguez Gonzales, S. (2018). El concepto de subordinación en el marco de la economía colaborativa. En Impacto laboral en las redes empresariales. Granada.

Rodriguez Marín, S. (s.f.). Los modelos colaborativos y bajo demanda en plataformas digitales. España: ADIGITAL. Recuperado de: https:// www.fidefundacion.es/attachment/810605/ (mayo de 2019).

Slee, T. (E. Iriarte, trad.). (2016). Lo tuyo es mío. Contra la economía colaboratina. 1. ${ }^{\text {a }}$ ed. España: Penguin Random House Grupo Editorial.

Supiot, A. (T. d. Rubini-Blanco, trad.). (2008). Derecho del trabajo. 1. ${ }^{a}$ ed. Buenos Aires: Heliasta.

Todoli Signes, A. (2017). El trabajo en la era de la economía colaborativa. Valencia: Tirant To Blanch.

Ugarte Cataldo, J. L. (2011). El Nuevo Derecho de Trabajo. Chile: Abeledo Perrot Legal Publishing.

Vallecito Gámez, M. R. (s.f.). Economía Colaborativa y laboralidad: los cabos sueltos entre el vacío legal y la dudosa legalidad. Recuperado de: https://www.ilo.org/wcmsp5/groups/public/--europe/---ro-geneva/---ilo-madrid/documents/ publication/wcms_615487.pdf

Sentencia del Tribunal de Justicia Europeo. (2017). Recuperado de: http://curia.europa.eu/juris/ document/document.jsf;jsessionid =ED82 E099C30864309175BC46A50ED074?text $=$ \&docid $=198047$ \&pagelndex $=0$ \&doclang $=$ $E S \&$ mode $=I$ st $\&$ dir $=\& O c C=$ first $\&$ part $=1 \& \mathrm{c}$ id $=5170896$ 